\title{
Block chain for Tertiary Education
}

\author{
Y.V.R.Naga Pawan', K.Bhanu Prakash ${ }^{2}$ \\ ${ }^{1}$ Department of CSE, Anurag Engineering College, Ananthagiri (V\&M), Suryapet(Dist), Telangana, INDIA. \\ ${ }^{2}$ Department of CSE, Koneru Lakshmaiah Education Foundation, Vijayawada, AP, INDIA. \\ ${ }^{1}$ ynpawan@gmail.com \\ 2drkbp@kluniversity.in
}

\begin{abstract}
Tertiary Education is expanded exponentially over a decade, in India. The statutory authorities are striving for inducing the quality in tertiary education through Outcome Based Education as one of the major transformations. The phoney credentials are culminating the initiatives and affecting the right spirit of transformation initiatives. Blockchain, a disruptive technology, with its public, secure and unique distributed ledger brings transparency to the details of students, faculty and Institutions, enabling the stakeholders and statutory bodies like AICTE, UGC, affiliating university to access genuine information from the data stored in the blockchain.
\end{abstract}

Keywords: Outcome based Education, Blockchain, Tertiary Education.

\section{Introduction}

The globalized industry is drastically changing its shape. It is the time for fourth industrial revolution i.e., Industry 4.0 (I40). The disruptive technologies like Artificial Intelligence (AI), Robotics, 3D Printing, High Speed Travel, extra-terrestrial colonization etc., are going to change the world and also population way of living. It is challenging for HR Managers to hire employees with high qualification and smart skill set along with self-engaging skills. If I40 employs human force as required, new performance management tools are required in coming years for assessment. To caters, the demand of I40, the Technical Education (TE) needs to transform transiently which enables the upcoming engineering graduates are highly employable. The demand for quality engineering education may rise steeply.

\section{Higher Education in India}

The Higher Education Institutions (HEI) or Tertiary Education (TE) have to play a pivotal role, in building new India in imminent decades. Numerous

\section{Corresponding Author}

Y.V.R. Naga Pawan, Anurag Engineering College, Ananthagiri, Suryapet(Dist), Telangana, INDIA ynpawan@gmail.com

Engineering Institutions are producing a large of Graduates, Post Graduates and also Doctorates, these days. In Particular, the Engineering Graduates from Centrally Funded Institutions (CFIs) like Indian Institute of Technology (IITs), National Institute of Technologies (NITs), and State level Government Universities and Government Engineering College and a very few reputed Private Institutions are comfortably employable irrespective of the vacillations in the Industry. Majority of the Tertiary Technical Education (TTE) is imparted through SelfFinancing Private Institutions. As per statistics of All India Council for Technical Education (AICTE), a statutory body to oversee the Technical Education in India, reports that majority institutions are imparting low quality technical education which is not suitable for the current and future industry.

The Ministry of Human Resource Development (MHRD), Government of India (GoI), robustly believes that the institutions with well-established infrastructure and quality faculty impart quality education to the students. MHRD through AICTE instructing the Technical Education Institutions (TEI) to adopt Outcome Based Education (OBE) and insisting to get accreditation for the programs offered. Globally, it is evidenced that OBE enables the TEIs to transform Engineering Education to the necessities of Stakeholders.

\section{Outcome Based Education}

Outcome Based education is an educational theory Model based on goals (outcomes) for each part of an educational system. It is a student-centric learning model. In OBE, the program created with an expected outcome as per the requirements of the stakeholders. The curriculum is designed with the aim of equipping learners with the knowledge, skills and orientations needed for success after leaving the institution. The OBE model measures graduates' progress from the parameters, which are

$$
\begin{array}{ll}
\text { - } & \text { Program Educational Objectives (PEO) } \\
\text { - } & \text { Program Specific Objectives ( PSO) } \\
& \text { Program Outcomes (PO) }
\end{array}
$$


- $\quad$ Course Outcomes $(\mathrm{CO})$

Various direct and indirect assessment tools are used for measuring the COs, POs, PSOs and PEOs.

The role of the faculty is critical in the implementation of the OBE (Harden RM 2007). The faculty adapts into instructor, trainer, facilitator, and/or mentor based on the outcomes targeted. It is obvious many times that great teaching has positive impact on student outcomes. The faculty must choose apt teaching methods to enhance the students' learning experience. This is conceivable, if the faculty are constantly learning through Continuous Quality Improvement (CQI). As a part of CQI, various professional development opportunities are provided to the faculty. The faculty perspective towards OBE will improve when they are encouraged to improve qualification and supported to participate in seminars/workshops, presenting papers in the reputed national/ international conferences/journals, writing books chapters and books, conducting seminars/workshops/ conferences to yield better results. The credentials gained through CQI helps them to beget momentum in the implementation of OBE and also its quality.

The implementation of OBE relies on consensus, trust and transparency. It is a dissuasive challenge for TEIs to comply with the rules and regulations of all regulatory bodies and to prove the integrity of data relating to students, faculty and institutes, and to prevent data breaches.

\section{A. Benefits}

The implements of OBE benefits the TEIs in terms of

- Clarity in outcomes to be achieved,

- Flexibility in choosing diversified teaching methods,

- Comparison in terms of implementation of OBE, credits awarding leading to movement of students across the Institutions to Institutions or Institutions to Organizations,

- Involvement of students in learning and other stakeholders to uphold quality of Technical Education.

B. Advantages

The advantages of OBE include

- Relevance

- Controversy

- Acceptability

- Clarity

- Provision

- Accountability

- $\quad$ Self-Directed Learning

- Flexibility

- Guide for Assessment

- Contribution to curriculum planning

- Curriculum evaluation

- Lifelong learning

C. Disadvantages

The disadvantages of OBE are:

- Education should be open ended but not constrained by outcomes.

- $\quad$ Emphasis on Attitude and values
- Learning by discovery or Inquiry is least preferred

- No emphasis for slow learners.

- Possibility of vague conceivable outcomes by implementers

- Usage of specialised resources for struggling students, which is cost effective.

- Drastic increase in the workload of a faculty

4. Issues in Implementation of Transformations in TIs The transformation efforts from statutory bodies like UGC, AICTE, PCI, affiliating Universities for implementation of OBE and various exemplary reforms (UGC, 2016) for improvement in tertiary education are waning (Chaitanya Mangure, 2019) (Jaydeep, 2016) (Outlook Web Bureau, 2018) (Akshaya Mukul, 2019) (TNN, 2019) (TNIE, 2019) (VRC Phaniharan, 2019) due to following reasons:

- Unethical practices in Continuous Internal Evaluation and assessment.

- Fake Degree awarded by unapproved institutions.

- Equal treatment for Accredited and non-accredited programs by affiliating university while awarding the degrees.

- No specific recognition for the Graduates who have degrees for accredited programs.

- Faculty with specious degrees from Graduation level to Doctoral level.

- Publications in non-indexed and predatory journals.

- Claiming of spurious consultancy services by faculty.

- Unauthentic claim of placements in the Institutions.

- Showcasing fallacious credentials of the students in the Institution websites.

\section{Blockchain - A Technology for Transparency}

Blockchain has its reputation in view of Bitcoin and its predominance in digital security limits, doable in understanding, trust, transparency and wellbeing.

In the simplest of terms, a blockchain is a time-stamped series of immutable data recording that is managed by a cluster of computers not owned by any single entity. Each of these data blocks (i.e., blocks) is secured using cryptographic principles (i.e., chain) and linked to each other.

There is no central authority in the blockchain network it is the very definition of a democratized system. Since it's a shared and immutable ledger (Crosby et.al, 2016), it's open to anyone and everyone to see the information in it. Therefore, by its very nature, everything that is built on the blockchain is transparent and all involved are responsible for their actions.

Blockchain was invented in 2008 by a person (or group of people) who used the name Satoshi Nakamoto (Nakamoto, 2008) to serve as the cryptocurrency bitcoin's public transaction ledger. It is unknown the identity of Satoshi Nakamoto. The blockchain's invention for bitcoin made it the first digital currency to solve the problem of double spending without the need for a trusted authority or central server. The bitcoin design has inspired other applications and blockchains are widely used by cryptocurrencies that are readable by the public. 
Blockchain has two basic characteristics. The blockchain is open to the public. It can be viewed at any time by anyone because it resides on the network and not within a single institution charged with maintaining and keeping records. Blockchain has also been encrypted as it uses public and private key encryption to ensure its security. Figure 1, represents the general idea how this technology works.

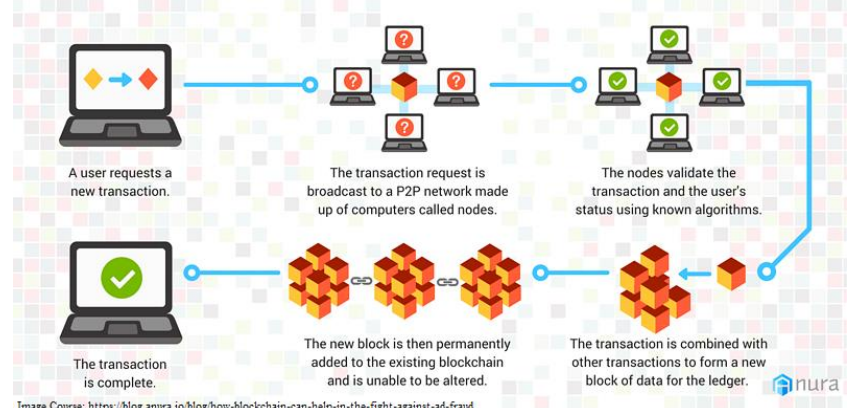

The bitcoin scheme places transactions in groups called blocks and then links these blocks through what is called blockchain. In one block, transactions are considered to have occurred simultaneously. These blocks are connected in a linear, chronological order to each other (like a chain), with each block containing the prior block's hash.

\section{A. A New Digital System}

Blockchain establishes the new era of the digital economy, there are seven design principles for creating software, services, business models, markets, organizations, and even governments on the blockchain (Tapscott et.al, 2016). Blockchain technology can be integrated into multiple areas. The details are as follows:

- Networked Integrity

- Distributed Power

- Value as incentive

- Security

- Privacy

- Rights Preserved

- Inclusion

B. Types of Blockchain from Technological perspective

There are predominantly three types of blockchains (Buterin, 2015) emerged technological standpoint, after Bitcoin introduced Blockchain to the world.

- Public blockchain

- Private blockchain

- Federated blockchain or consortium blockchain

1) Public blockchain: It is a permission less blockchain, the users may read, write or participate with a public blockchain. It is a decentralised, distributed, no one has control over the network. It is immutable, once data written cannot be changed, after the data is validated. The new blocks can be added but the blocks cannot be removed or replaced. The public blockchains are backed by consensus based algorithms like Proof of Work (PoW) and Proof of Stake (PoS). Bitcoin is example of public blockchain.

2) Private Blockchain: It a permissioned blockchain. The permissioned networks imposes restrictions, who participates in the network. The write permissions are kept with one organization or institution, whereas the read permissions are public or restricted to an arbitrary extent. Multichain is an example for private blockchain.

3) Federated or Consortium Blockchain: Federated blockchain is partly private. The consensus process is controlled by preselected set of nodes. The right to read the blockchain may be public, or restricted to the participants. Consortium blockchain platforms have many of the same advantages as a private blockchain, but operate under the leadership of a group instead of a single entity. Ethereum is an example for this type of blockchain.

\section{Types of Blockchain from Business perspective}

From the business perspective, there are two types of blockchains, open blockchain and closed blockchain.

1) Open Blockchain: A public network that keeps a record of operations unchanged. Anyone can publish a transaction by adhering to a set of published regulations and participating in the network. Open blockchain with Artificial Intelligence (AI) may bring rapid changes in the business models and also evolving new business applications for future.

2) Closed Blockchain: A private network that retains a shared transaction record. Only those with authorization can access the network, and administrators can edit transactions. Private or Federated Blockchain are closed blockchains. These blockchains uses predefined environment ie an enterprise based solution. The changes are allowed to the business environment but an optimization of process is beneficial. The innovation is incremental. These blockchains are used by known players in the Industry. It puts in one blockchain solution different known players together. The players already know each other, so confidence is not an issue. Optimization is possible because everybody has the same data at the same time.

\section{Application of Blockchain}

Digital payment systems, smart contracts, Insurance, crowfunding are the popular financial applications of blockchain whereas the applications like decentralized government services, decentralized storage, decentralized Internet of Things (IoT).

\section{Blockchain in Tertiary Education Institutions}

Blockchain is the hope for TEI's as the proprietary data management become complex due to manual process. In majority of TEI's, the data is categorised as Students, Faculty and Institutional.

As the blockchain promises trust and immutable nature of its transaction records, the credentials of the students, Faculty and Institution are stored permanently in a uniform structured in the blockchain. The up to date stored data is accessible to all the stakeholders, statutory bodies, accreditation agencies etc. Thus reducing manual record verification by third party agencies and continuous 
Journal of Engineering Education Transformations, Volume 33, January 2020, Special issue, eISSN 2394-1707 functioning of Institution Management Information System or record-keeping system in the TEI.

\section{A. Student Credentials}

The student credentials relating to courses, projects, credits, scores, grades, credits transferred through MOOCs, information of accreditation programs etc., are converted as digital credentials in the blockchain and shared to the Universities for making the credentials authenticated, tamper proof, legally valid and creates unified view and shares the same to the TEI. The unified view of the student's credentials are publicly accessible to all the stakeholders such as parents, other universities, employers etc. This avoids credential tampering. This is also enables transition of the students from Institution to another Institution, globally. It is easy for the TEIs to track their alumni and their credentials earned after leaving the Institution.

\section{B. Faculty Credentials}

The TEI is transformed into an industry, thus began the unqualified faculty taking the teaching positions through fake degrees and fake credentials, as the manual verification is inefficient and corrupted shoving the TEI industry into a quagmire.

The faculty credentials like qualifications and their degrees, courses learnt thorough MOOCs, professional memberships, research publications like (Prakash KB et al, 2015) (Prakash KB et al, 2016), patents, books written, consultancy services, conferences / seminars chaired and many other academic and financial credentials are placed in the blockchain as permanent records which are accessible to all the statutory bodies and potential employers. Thus avoiding fake faculty with faulty claims. The genuine faculty will be exhilarated. Such faculty will be rewarded or incentivised or promoted for their authentic credentials available publically and with apposite recognition globally.

\section{Institutions Credentials}

Many TEIs are luring the students to take admission in their Institutions showcasing fake achievements and Institution credentials. By placing the Institution credentials such as statutory body approvals like AICTE / UGC / PCI approvals, affiliating university affiliations and their validity, accreditations details (NBA, NAAC etc), placements of the students, achievements in curricular and extra-curricular activities on the blockchain enables the stakeholders to access the genuine information. A quality OBE is implemented. As the data about students, faculty and institute is available in blockchain publically, this enables

- the statutory bodies to verify the norms followed and credentials possessed by the Institutions to grant approvals or affiliations,

- accreditation agencies to evaluate the Institution data and accord accreditation with less data collection and also without a Peer-Team visit to an institution, unless required, saving time and money.

\section{E. Identity Verification} Identity verification is one of the Perennial problem in the TEIs. This provides a window of opportunity for data tampering. With blockchain student, faculty, institution credentials are verified and approved once through Proofof-Work (PoW), Proof-of-Stake (PoS) and Proof-ofImportance (PoI), the consensus algorithms for blockchain.

\section{Conclusion}

Blockchain is a disruption technology which changes the organization model in TEIs. It serves a solution to deal with fake TEIs, Degrees and Faculty. A federated blockchain is apposite. The readily available public ledger makes the verification faster and robust with less human intervention in a less time and also ensures transparency of data pertaining to the students, faculty and TEIs for the stakeholders through secure platform. Blockchain facilitates the Institutions for quality implementation of OBE. Blockchain allows statutory bodies to grant swifter approvals or affiliations, faster evaluation of Institutions or Programs specific for accreditation by accreditation agencies.

\section{References}

Nakamoto, Satoshi (2008), "Bitcoin: A peer-to-peer electronic cash system".

Harden RM (2007), Outcome-based education - The ostrich, the peacock and the beaver, Medical Teacher, 29(7), pp 666-71, Sep 2007 doi://10.1080/01421590701729948.

Tapscott, Don and Tapscott (2016) ,Alex. Blockchain Revolution: How the Technology Behind Bitcoin Is Changing Money, Business, and the World. Penguin.

Crosby, Michael et al. (2016), "Blockchain technology: Beyond bitcoin". Applied Innovation, pp. 6-10.

Prakash, K.B., Dorai Rangaswamy M.A. (2016), Content Extraction of biological datasets using soft computing techniques, Journal of Medical Imaging and Health Informatics, 6(5), pp 932-936.

Prakash, K.B., et al (2015), Information extraction in unstructured multilingual web documents, Indian Journal of Science and Technology, 8(16), pp 1-8.

\section{Web References}

Buterin, Vitalik. On Public and Private Blockchain. Aug. $6^{\text {th }} 2015$. url: https://blog.ethereum.org/2015/08/07/onpublic-and-private-blockchains/. Last Accessed on 06.11.2019.

BlockchainHub. Types of Blockchain. url: https://blockchainhub.net/blockchainsin-

general/. Last Accessed on 06.11.2019

Chaitanya Mangure (2019) Engineering for Rs 75,000, Law Certificate for Rs 2 Lakh: How the Fake Degree Market Flourishes url: https://www.news18.com/news/india/ engineering-for-rs-75000-law-certificate-for-rs-2-lakhhow-the-fake-degree-market-flourishes-2289559.html Last Accessed on 06.11.2019

Jaydeep (2016), Educational Frauds In India : Fake 
Universities url: https://www.mapsofindia.com/myindia/government/educational-frauds-in-india-fakeuniversities-and-forged-degree-certificates Last Accessed on 06.11.2019

Outlook web Bureau (2018), Pan-India Fake Degree Racket Busted, Accused Sold 50,000 Certificates Of Universities, Schools url: https://www.outlookindia.com/website/story /pan-india-fake-degree-racket-busted-accused-sold-50000-

certificates-of-universit/307562, Last Accessed on 06.11 .2019

UGC (2016), public Notice on Fake Universities By UGC url: https://www.ugc.ac.in/pdfnews/6743812_UGC-Fakeuniversities-list.pdf, Last Accessed on 06.11.2019.

Akshaya Mukul (2011), Engg colleges fake faculty's qualification for govt sanction url: https://timesofindia.indiatimes.com/india/Engg-collegesfake-facultys-qualification-for-govt-

sanction/articleshow/9007159.cms, Last Accessed on 06.11.2019.

TNN (2019), JNTUH to tighten the noose on fake faculty. url: https://timesofindia.indiatimes.com/city/hyderabad/ jntuh-to-tighten-the-noose-on-fake-faculty/articleshow/ 71016903.cms, Last Accessed on 06.11.2019

TNIE( 2019), Fake-faculty scam returns to haunt JNTU-H after 4 yrs url: http://www.newindianexpress.com/cities/hyderabad/2019/ aug/04/fake-faculty-scam-returns-to-haunt-jntu-h-after-4yrs-2013904.html, Last Accessed on 06.11.2019

VRC Phaniharan (2019), Degrees for sale: Fake faculties thrive in TS, url: https://www.thehansindia.com/telangana/degrees-for-salefake-faculties-thrive-in-ts-558901, Last Accessed on 06.11.2019. 